\title{
Introducing Methods Moment
}

Nina Exner, PhD, MLS

Assistant Professor / Research Data Librarian

Virginia Commonwealth University

Richmond, VA

A new column is being introduced to Hypothesis, called Methods Moment. As the name suggests, the column will focus on brief discussions of methodological topics for librarians who are doing, or wish to do, their own research. Librarians typically do not delve deeply into research class in library school or hear research concepts sprinkled throughout their library school courses. Nevertheless, librarians often want or need to do research! Regrettably, much of the methods literature is not geared towards practitioner-researchers who want to start or expand their research career. To support librarians new to research or expanding their interests, Methods Moment wants to introduce various methods for librarians to explore and apply.

Methods Moment aims to give short, practical introductions to various methods of use to medical librarians. Our goal is to help librarians to familiarize themselves with various methods, and to think about which methods will or won't work for answering their own research questions. Each column should serve the goal of increasing the breadth of methodological concepts that readers are familiar with.

Submissions to the column are subject to blind peer review. If prospective authors would like advice on developing an idea into a column, column editor Nina Exner, nexner@vcu.edu, will give guidance and feedback to authors about style and scope-fit. The Hypothesis journal editor(s) have final discretion over the acceptability of any column submitted.

\section{COLUMN FORMAT}

Methods Moment columns should be 1,250-2,000 words per column. They should be written in a casual, practical style. A given column will not aim to be comprehensive of a topic; instead, it should give enough detail to familiarize the reader with the topic, help them imagine the topic's practical application in health librarian research, and lead interested readers to explore further.

The structured abstract serves as a place for librarians to determine whether a given method is relevant to them at all. It covers:

- Objective: What is this method intended to do or discover?

- Approach: How does the method work? What describes the unique features or technique of this method?

- Data type(s) used: What data goes into this method? Is it interviews, health records, national surveys, big data, etc.? 
- Strengths: What is uniquely good about this method?

- Limitations: What are common limits or issues to be aware of when using this method?

Each Methods Moment column is intended to provide (1) an overview of the topic; (2) a practical example or illustrative mini-case; and (3) resources for further exploration.

- Overview: The overview should include a short definition or description of the method or topic. It will then explain the method or topic, its purpose or role in producing research findings, and why it might be of interest to health science librarians in their research. The overview should stand on its own for readers who are curious but do not want to explore further.

- Example: The example section should illustrate how the method or topic plays out during research in practice. The example might be a narrative description of the topic in action, a personal experience of application, or a mock case study describing an imaginary scenario of when and how the topic would be applied.

- Resources: The resources section should point readers to places for further exploration and self-guided learning. This should be a curated list of materials for interested learners. Wherever possible, if learning materials exist, they should be pointed to in the resources rather than trying to include instructional content in the overview. Most of the resources should be freely accessible, rather than subscription resources with limited availability.

However, alternative formats may be considered! Good alternative formats could include compare-and-contrast between two related methods, brief reports of methods focusing on ideas for self-guided exploration, or summaries of discussion events among librarians who use a certain method. Creative approaches to addressing the needs of practitioners new to research are encouraged. 